\title{
A Review on WMSN (Wireless Medical Sensor Networks) for Health Monitoring Systems
}

\author{
Harshal Rahangdale ${ }^{1}$, Prof. Nekita Chavhan ${ }^{2}$, Pritesh $\mathrm{Ade}^{3}$ \\ ${ }^{1}$ Department of Computer Science and Engineering G.H. Raisoni College of Engineering, Nagpur, India, \\ ${ }^{2}$ Assistant Professor Department of Computer Science and Engineering G.H. Raisoni College of Engineering, \\ Nagpur, India, ${ }^{3}$ Cardion Technologies Private Ltd. \\ ${ }^{1}$ harshal.rahangdale.cs@ghrce.raisoni.net, ${ }^{2}$ nekita.chavan@ raisoni.net, \\ ${ }^{3}$ pritesh.ade@cardiontechnologies.com
}

\author{
Article Info \\ Page Number: $48-53$ \\ Publication Issue: \\ Vol 71 No. 1 (2022)
}

\begin{abstract}
Wireless Medical Sensor (WMSN) networks have become a great technology to allow for the best kind of applications. Recent developments at WMSN have helped to create a comprehensive health monitoring concept in both home and hospital care settings. Current technological advances in sensors, integrated dynamic circuits, and wireless connectivity have allowed the event of smaller, heavier, less expensive, and clever body sensors. These modules are able to hear, process, and communicate with one or more important signals. In addition, they will be used on local wireless networks (WPANs) or wireless nerve networks (WBSNs) to monitor health. Many studies are being conducted and/or ongoing to develop flexible, reliable, secure, realistic, and effective WBSNs for health applications. The importance of wireless nerve networks in health surveillance is constantly evolving, due to the growing need for safety and security in cities. The rapid development of wireless technology has significantly aided the advancement of monitoring systems that have been developed through the combination of wireless sensors. The wireless health monitoring introduces emerging innovations with significant advantages when compare to conventional communication networks, which has the advantages of lowering the cost of installation and maintenance while increasing the effectiveness of the health monitoring system in the field of medicine.
\end{abstract}

Keywords: - WMSN, Sensors, Circuits, Power-efficient, Communication.

\section{Introduction}

The application of technology to enhance one's standard of living is now becoming increasingly common in modern civilization. Technology that is geared towards increasing one's personal Quality of Life (QoL), such as the Internet of Things (IoT), is referred to as "the Internet of Things" (IoT). It is necessary to monitor the physiological parameters of the patient on a regular basis in a medical healthcare monitoring system. The fact that ward evaluation does not typically include continuous physiological parameter monitoring means that patient recurrence is relatively uncommon. Because of its excellent technology, the outpatient monitoring system has made a substantial contribution to the hospitality industry's overall quality. 
The Internet of Things (IoT) in healthcare refers to a range of low-cost devices (wearable, implanted, and environmental) that enable elderly people to receive medical treatment from any location, at any time, without having to travel. A temperature controller and a heart rate sensor are coupled together in addition to monitoring the patient's condition. In addition to providing medical services such as health monitoring and memory enhancement, wearable monitoring network systems can also be used to communicate with healthcare practitioners in an emergency situation via SMS or GPRS. Researchers come up with a solution that is composed mostly of off-the-shelf physical devices and that comprises modular sensor blocks as its building blocks.

To transmit information, modern medical equipment makes use of wireless technology, which is also known as radio frequency technology (RF technology). Anyone can access the information in real time and it provides excellent accuracy while eliminating the need for handicrafts. New and innovative ways are frequently discovered on wireless networks that employ Wi-Fi or Bluetooth and that are capable of being upgraded. Construction, resources, industry, home, shipping, and climate-change systems are all being redefined by smart areas. Smart areas include: Here, we will look at the parameter delivered again with an increased number of extra items, as well as the procurement of these homecoming initiators for the purpose of development and marketing of their goods in this programme. WMSN contains the ability to communicate emotions, if necessary, to human body system programmers and the health status of the observer. These autonomous devices, also known as nodes, communicate with other nodes and indeed the WMSN system. Great amid new challenges, such as determining the number of elderly people and collecting money from health-care expenses.

The development of health-care systems to control health prior to disease, as well as the determination of how diseases occur and the solutions to these problems are all possible. A wide range of potential health-care applications are provided by WMSN, ranging from monitoring physical symptoms to performance monitoring and medicine distribution, among other things. Chromium disease patients can be monitored continuously in their own homes, not by providing them with medical information, but by monitoring their environment in real time, according to the National Institute of Health. Its purpose is to establish communication between various WBNs that monitor or provide information about a certain patient and/or other surrounding sensors. If the terms Wi-Fi, Bluetooth, or $394 \mathrm{Zig}$ Bee are applicable, this function is not being fully leveraged by the company; therefore, it is considered underutilized. As an illustration, consider the work of Gupta and colleagues. They can cause falls or give people Alzheimer's disease, autism, dementia, or tracking devices for the Global Positioning System in situations where they create a platform for data exchanges, storage, search, detection, and analysis of data from them. They can also cause falls or give people autism, Alzheimer's disease, dementia, or tracking devices for the Global Positioning System in situations where they create a platform for storage, data exchanges, detection, analysis and search of data from them.

There have always been wireless products available that may be used to monitor sleep quality, scent, breathing, and bruxism, among other things. Wireless Body area Network (WBN) is one of the most extensively used terms in the termination industry, and it is short for Wireless Body Area Network. It is possible to use these sensors to detect that however much blessed is being created, as well as levels of blood sugar, mobility, heart rate, body temperature, brain signals, and so on. In the not-too-distant future, the wearable networks of networks will be separated into two categories: the most entertaining sensors and the least entertaining sensors. The research was carried out with the help of Wearable Wireless Sensor Networks 
(WWSN). Located within the health care facility or hospital is a safe place where patients can check their pockets, and so on. WN (Wireless Personal Area Network) is another network that has been developed to allow you to track patient performance from time to time according to the Electrocardiogram, EEG, and GSR data collected.

The following are some of the advantages of the prototype described above. It allows clinicians to monitor clients even though they're not physically available in front of a connected medical monitoring station, which is the first and most important benefit. In particular, when medical personnel are only sufficient to support immediate care, this is critical to remember. Patient monitoring outside of typical healthcare situations (for example, at home) is a technology that has the potential to increase access to care while also reducing health care expenditures. The primary function of this gadget is to monitor and maintain a person's core temperature and transmit that information to a doctor via radio frequency transmission. This project makes use of the Iot technology to monitor many indicators related to the patient's health and wellness. Patients' real-time health parameters are communicated to the cloud through the use of Internet access in the health monitoring system, which is based on the Iot technology project and access the Internet of things project. All of these characteristics are transferred to a remote Network location where they can be viewed by users all over the world. The Internet of Things (IoT) patient monitoring system employs three sensors.

In designed to motivate continuous monitoring and verification of a man's health state and interchange it through long distance connection, a remote geographically located system is used. As of right now, the medical atmosphere in developing countries permits individuals to personally attend for regular medical examinations or to be admitted for long-term observation and monitoring. The system is rigorous, and it takes a long time to complete. Patients can now manage their development are as follows from any location and at any time thanks to the advancements in remote sensor technology.

\section{Review of Literature}

It has been stated by the authors in [1] that dynamic routing or WSN trusted mechanisms should be examined in perspective of WMSN operational circumstances, network structure, and resources that are not functioning properly. Like the WMSN system, as stated in the ReTrust paper, a straightforward and antiattack system is being developed in parallel. It is possible for a single WMSN to have hundreds of patients in a single body network. All BSNs contain a large number of SNs (Sensory Sensors), which are responsible for sensing and controlling the body's natural impulses and functions. Sensitive networks are equipped with high-quality hardware components that are capable of coping with a wide range of security challenges. Energy barriers, in addition to sensor network (BSN) networks, are also present, and these are substituted by batteries that comprise lithium iodide cells.

When attempting to obtain statistically significant results, the author of [2] proposes a more efficient procedure. Collection of data, energy consumption, surveillance systems, and network management have all been examined, as well as common disaster detection, such as forest fires, control equipment, and speeding via some of WSN's applications, among others. Farming encompasses a broad range of application areas in which the challenges of implementing WSN and WSAN are significant. WSN functions consecutively in a separate scenario with an unique directed wireless directory, as opposed to other types of networks.

An electronic surveillance system for regulating bodily parameters such as blood pressure, temperature, and respiration was proposed by the authors in [3]. An example of a gateway might be a service that connects to other services, management, or a platform designed exclusively for something like the residential healthcare 
delivery system. Improving the integration of WSNs and social networking networks, as well as offering a somewhat more adaptable decision-making mechanism and compact data are all goals of the project.

In [4], the author advocated the use of a wireless sensor nodes (WSN). In health- related applications, performance analysis is performed via a system of wireless sensor nodes. Patient correspondence, highquality guidance, and the patience of a firm specialist are all examples of the current pains. The requisite safety expertise of the individuals who will be collecting the information can be a questionable problem.

The author of [5] proposed a comprehensive overview, including devices and communication equipment technology, network infrastructure, critical risks and open research challenges related to SHM's WSN, and other relevant topics. In addition to free key elements known as single-sensory systems, which include computing systems and medical examination processes, the conventional SHM system also includes other free key elements.

The author of [6] presented a patients patient monitoring system that was developed and tested by the WSN method using the Big-zee protocol, which was developed and tested by the WSN protocol. Health care is a type of individual monitoring that is used to detect changes in the state of one's health that are caused by certain factors or conditions. The process of health monitoring concludes with a session in which you answer questions about your medical history, such as whether you smoke or the various health consequences of chemicals.

As stated by the author of study [7], the use of multiple wireless sensors to demonstrate how and where to monitor the numerous medical parameters of users using different sensors such as a temperature sensor, blood pressure sensor, ECG sensor and pulse sensor can be demonstrated using different sensors. It emphasizes the use of multiple wireless sensors to study how you may assess different health issues for users utilizing different sensors such as a pulse sensor, a temperature sensor, and an ECG sensor, which could be used to effectively monitor heart rate, among other things.

Several wearable, transportable, inexpensive, and compact sensors were described by the authors in [8], all of which were intended to check the limitations of something like the internal organs. The goal is to integrate the long-distance and low-power consumption characteristics of LoRaWAN and ZigBee technologies. By utilizing ZigBee and LoRaWAN technology, we are prepared to communicate and present healthcare outcomes and RTL heard through distant troops to a group leader.

The concept of a system-building pill has been introduced by the authors in [9]. Specifically, we have recommended and visualized any use of Pill Sensing to monitor compliance to medicine, with the goal of improving patient outcomes. Pill Sense is indeed a wireless barrier with a long battery life and a precise system that is dependent on medicinal acquisition activities and has a long battery life.

As stated and described by the author in [10], communication and computer infrastructures and medical fields collaborating in order to provide a holistic perspective of smart health care that is dependable. Any use of wireless communication has expanded substantially as a result of its ease of use and efficiency for a variety of applications. WSNs are often terminated with a large number of sensors.

\section{Designing Factors and Discussion Related to Challenges}

Healthcare monitoring and surveillance systems based on the Internet of Things (IoT) constitute a framework of medical devices that may gather and share data among themselves in order to distribute a wide range of health care software and systems to patients. Healthcare practitioners are already accepting IoTbased wearable technologies to expedite the diagnosis and healing process of patients with chronic illnesses. 
However, there are some limitations, complications, and pertinent considerations that make it difficult to improve indefinitely in this area. As a result, the following are some of the obstacles and development issues that are addressed:

- With machine learning and artificial intelligence, it is possible to conduct multidisciplinary research and execution of analytical approaches that seem to be essential for the development of future smart healthcare, encompassing surveillance and decision assistance. Machine learning are currently the focus of a substantial amount of research efforts aimed at assisting decision-making. Such investigations offer significant potential for resolving a wide range of the issues that have been found. Recent advances in machine learning and artificial intelligence may not completely replace physicians, but they may enhance their ability to convey critical information that a patient needs to know and provide it in a short, easily comprehensible manner. Such innovations would not only improve decision-making assistance, but they would also maintain the integrity and dependability of all of the components that make up the smart healthcare monitoring ecosystem as a whole.

- With machine learning and artificial intelligence, it is possible to conduct multidisciplinary research and execution of analytical approaches that seem to be essential for the development of future smart healthcare, encompassing surveillance and decision assistance. Machine learning are currently the focus of a substantial amount of research efforts aimed at assisting decision-making. Such investigations offer significant potential for resolving a wide range of the issues that have been found. Recent advances in machine learning and artificial intelligence may not completely replace physicians, but they may enhance their ability to convey critical information that a patient needs to know and provide it in a short, easily comprehensible manner. Such innovations would not only improve decision-making assistance, but they would also maintain the integrity and dependability of all of the components that make up the smart healthcare monitoring ecosystem as a whole.

- Healthcare monitoring surveillance systems are essential for ensuring the validity and consistency of public health care information in the field. As a result of daily use, the accuracy and dependability mechanisms must be maintained, otherwise misleading information may have become imprecise and obstructive to patients. When it comes to remote healthcare monitoring systems, the Internet of Things (IoT) technology has a very strong vested interest, for example, in incorporating and interpreting numerous shapes of health records in professional decision-making processes. Physicians will be able to conduct a thorough assessment of each patient's health status, allowing them to provide more effective interventions. All of this has been accomplished through screening procedures, at which advancement accuracy has remained critical in order to save the patient's life.

- Smart sensors and devices that are capable of operating constantly on-site and consuming little energy are typical components of remote healthcare monitoring systems. It appears that the utilization of renewable energy for responsible health - monitoring techniques has emerged as a new problem. The Internet of Things has given researchers the ability to perform emerging breakthroughs in order to reduce power consumption of a large number of simultaneous smart sensors and devices. As a result, several protocols have been proposed, procedures, and strategies have already been demonstrated to reduce the total amount of energy required for network operation. Nevertheless, the volume of data created must also be managed in order to further reduce the amount of power required for interpretation and transmission of data.

- Healthcare monitoring and surveillance systems that are energy efficient can considerably reduce the 
amount of energy consumed in healthcare facilities, resulting in significant power savings for the facility. These methods have a positive impact on the environment as well, because they reduce electromagnetic interference.

Conclusion

A growing body of evidence about how remote health surveillance technologies may be used in healthcare is needed to inform strategic decisions, despite several observational data on the predictors of remote medical surveillance technologies. The potential for large-scale trials with creative features to possibly clarify the impact on the patient monitoring, satisfaction level, and operation costs exists as a consequence. A wide range of experience and competencies are still required in this rapidly evolving field for example, stronger emphasis on socio - economic status analysis methods of distant location patients' medical evaluate investment, on the condition of individuals and their perspective of healthcare monitoring as dynamic processes for growth and productivity, as well as on the continuation of cooperative results in the future. As a result, the underlying evaluations for a field's limitations and significance are largely clarified in this article.

\section{References}

1) Muhammad Shadi Hajar, M. Omar Al-Kadri, Harsha Kalutarage "LTMS: A Lightweight Trust Management System for Wireless Medical Sensor Networks" 2020 IEEE 19th International Conference on Trust, Security and Privacy in Computing and Communications(2020) .

2) Naila Nawaz Malik, Wael Alosaimi, M. Irfan Uddin, Bsader Alouffi, and Hashem Alyami "Wireless Sensor Network Applications in Healthcare and Precision Agriculture" (2020).

3) P. Karthikeyan, C. Ashwin, R. Sai Vishnu, N. Ravi teja4 "Electronic Vaccine Monitoring System" International Journal of Recent Technology and Engineering (IJRTE) ISSN: 2277-3878, Volume-8 Issue-6, (2020).

4) Shanthi Kumaraguru,Sathyabama, Dr.M.R. Ebenezar Jebarani, "Secured Human Health Monitoring Using Wireless Medical Sensor Networks" European Journal of Molecular \& Clinical Medicine ISSN 2515-8260 Volume 07, Issue 08, (2020).

5) Mohammed Abdulkarem, Khairulmizam Samsudin, Fakhrul Zaman Rokhani and Mohd Fadlee A Rasid, "Wireless sensor network for structural health monitoring: A contemporary review of technologies, challenges, and future direction" (2019).

6) T S Sollu, Alamsyah, M Bachtiar and A G Sooai, "Patients' Heart Monitoring System Based on Wireless Sensor Network" IOP Conf. Series: Materials Science and Engineering 336 (2018) 012009(2018).

7) Jigar Chauhan, Sachin Bojewar, "Sensor Networks based Healthcare Monitoring System" (2018).

8) Aashay Gondalia, Dhruv Dixit, Shubham Parashar, Vijayanand Raghava, Animesh Sengupta, "IoT-based Healthcare Monitoring System for War Soldiers using Machine Learning" International Conference on Robotics and Smart Manufacturing (2018).

9) Murtadha Aldeery, Richard P. Martin, Richard E. Howard, "PillSense: Designing A Medication Adherence Monitoring System Using Pill Bottle- Mounted Wireless Sensors" (2018).

10) Matthew N. O. Sadiku, Kelechi G. Eze, Sarhan M. Musa, Roy G. Perry, "Wireless Sensor Networks for Healthcare" (2018). 